\title{
Variación en las abundancias de Homocopris achamas (Harold, 1867) (Coleoptera: Scarabaeidae: Scarabaeinae) en el páramo de Rabanal, Boyacá-Colombia
}

\author{
Variation in the Abundances of Homocopris achamas (Harold, 1867) (Coleoptera: \\ Scarabaeidae: Scarabaeinae) in the Rabanal Paramo, Boyacá-Colombia
}

\author{
Oscar Felipe Moreno Mancilla ${ }^{a^{*}}$ \\ Fredy Molano Rendón ${ }^{b}$
}

Recepción: 27-abr-2015

Aceptación: 28-abr-2016

\begin{abstract}
Resumen
El conocimiento sobre los escarabajos coprófagos de los páramos en Colombia es insuficiente y limitado, pues estos ecosistemas han sido poco muestreados para el caso de este taxón. El artículo describe la variación de las abundancias de Homocopris achamas, única especie reportada de la familia Scarabaeidae en el páramo de Rabanal. Se capturaron un total de 46 individuos: uno en la cobertura de Bosque, cinco en el borde Bosque-Frailejonal, 22 en Frailejonal y 18 en Pastizal. De estos, el $36.4 \%$ fueron hembras y el $63.6 \%$, machos. La totalidad de los individuos fueron colectados durante la época lluviosa. Se encontraron diferencias significativas en las abundancias de H. achamas en las diferentes coberturas vegetales presentes en el páramo, sugiriendo mayor preferencia de la especie por las coberturas de frailejonal y pastizal que por las coberturas de borde e interior de bosque.
\end{abstract}

Palabras clave: Escarabajos coprófagos, Alta montaña, Páramo de Rabanal.

\begin{abstract}
The knowledge about dung beetles in Colombian paramos is insufficient and limited, due to that these ecosystems have been poorly sampled for Scarabaidae family case. This paper aims to describe the variation in the abundances of Homocopris achamas, unique species of the family Scarabaeidae reported in the Rabanal paramo. 46 specimens were collected, from which one was captured inside the forest, five in the frailejonal-forest edge, 22 in frailejonal and 18 in grassland. $36.4 \%$ of them were female and $63.6 \%$ were male. All specimens were collected during the rainy season. Significant differences in the H. achamas abundances were found in the different vegetables covers present in the paramos, suggesting that H. achamas prefers frailejonal and grassland covers more than the forests edge and interior covers. prefers frailejonal and grassland covers more than the forests edge and interior covers.
\end{abstract}

Key words: Dung Beetles, Highland, Rabanal Paramo.

\footnotetext{
${ }^{a}$ Biólogo. Universidad Pedagógica y Tecnológica de Colombia. Grupo de Investigación Sistemática Biológica SisBio. Grupo de estudio en biología evolutiva y epistemológica BIEVEP, Tunja, Boyacá, Colombia.

*Autor de correspondencia: felipemmancilla@gmail.com

${ }^{b}$ Profesor Escuela de Ciencias Biológicas. Universidad Pedagógica y Tecnológica de Colombia. Laboratorio de Entomología UPTC. Grupo de Investigación en Sistemática Biológica SisBio. Museo de Historia Natural Luis Gonzalo Andrade. Tunja, Boyacá, Colombia
} 


\section{Introducción}

Los páramos neotropicales son ecosistemas con condiciones climáticas, de suelo y altitud peculiares, las cuales hacen de ellos conjuntos de condiciones ambientales particulares que se reflejan en una composición florística y faunística singular y rica en endemismos; en especial, son fuente de importantes servicios ambientales, entre los que se destacan el albergue de una biodiversidad única y los procesos de almacenamiento y regulación hídrica, lo cual los convierte en biomas de importancia estratégica para la sociedad [1]. A pesar de esto, y de ser declarados prioritarios para su protección y conservación en Colombia (Ley 99 de 1993), son uno de los ecosistemas más afectados por la intervención antrópica, principalmente por los cultivos de papa y la ganadería [1].

En el caso del páramo de Rabanal, ubicado en el complejo Rabanal-río Bogotá, en el altiplano cundiboyacense, el reemplazo de ecosistemas naturales por pastos y cultivos de papa supera ya el $45 \%$ de su superficie total, dejando aún algunos fragmentos de ecosistemas naturales propios de la región altoandina en diferente estado de intervención y de alta importancia para la estabilidad hídrica regional y la conservación de la biodiversidad [1].

Los artrópodos son uno de los grupos animales que mejor se adaptan a ambientes extremos como el páramo; presentan una importante diversidad y riqueza [2], producto de la heterogeneidad espacial típica de ambientes de alta montaña tropical, la cual genera microhábitats con potencial para ser colonizados por insectos, arácnidos, miriápodos y otros [3]. Diversos estudios acerca de las comunidades de artrópodos de páramo han sido realizados [2, 4-13, entre otros], en los que se identifican los grupos de artrópodos asociados tanto a comunidades vegetales como a unidades de suelo en páramos de la cordillera oriental; además de la descripción de especies nuevas para los órdenes Odonata y Lepidoptera.

En particular, el conocimiento acerca de los escarabajos coprófagos de los páramos en Colombia es insuficiente y limitado; estos ambientes son uno de los ecosistemas que se consideran poco muestreados, para el caso de este taxón [14]. Dentro de los estudios llevados a cabo se encuentran los realizados por Escobar [15], que cita seis especies en alturas mayores a $2250 \mathrm{~m}$; Medina et al. [16], quien adicio- na dos especies más por encima de los $2800 \mathrm{~m}$, y Martínez-Revelo \& Lopera [17], quienes reportan ocho especies de escarabajos coprófagos para los páramos de Nariño, incluyendo Homocopris achamas.

En los páramos de Boyacá, el estudio de escarabajos coprófagos es escaso, a excepción de los trabajos realizados por Molano \& Medina [18] y Molano \& Parrales [19], en los que se describen dos nuevas especies del género Scybalocanthon, registradas entre 2600 y $3000 \mathrm{~m}$, en los municipios de Arcabuco y Miraflores, respectivamente; además de unos cuantos reportes de la especie Homocopris achamas para el municipio de Sutamarchán, a 2800 $\mathrm{m}$, en la vereda Carrizal, y a $3220 \mathrm{~m}$, en la vereda Alto del Ermitaño, cuyos ejemplares están depositados en el Instituto Alexander von Humboldt (IAvH). Lo anterior evidencia la necesidad de conocimiento acerca de la presencia de estos insectos, de sus interacciones y dinámicas en los páramos, ya que esta información puede ser útil para planes de manejo y conservación de estos ecosistemas, dado el importante rol de estos escarabajos en el reciclaje y circulación de nutrientes, e incluso en la dispersión de semillas [15].

El presente trabajo tiene como objetivo describir la variación de las abundancias de Homocopris achamas, única especie registrada en el páramo de Rabanal por encima de los $3345 \mathrm{~m}$, en las diferentes coberturas vegetales (Bosque, borde Bosque-Frailejonal, Frailejonal y Pastizal) presentes en este lugar.

\section{Metodología}

\section{1. Área de estudio}

El páramo de Rabanal hace parte del complejo de páramos Rabanal-Río Bogotá; está ubicado en la cordillera Oriental, bajo la jurisdicción del municipio de Ventaquemada, en el departamento de Boyacá, Colombia $\left(05^{\circ} 24^{\prime} 42,28^{\prime \prime}\right.$ N y $73^{\circ} 32^{\prime} 41,87^{\prime \prime}$ O) (figura 1). El Páramo de Rabanal tiene un área total de 5200 ha; inicia sobre los $3030 \mathrm{msnm}$ y presenta altitudes máximas de hasta $3400 \mathrm{~m}$ [1].

Rabanal presenta un régimen de distribución de lluvias de tipo bimodal, cuyos períodos de mayor pluviosidad se presentan entre marzo a julio y octubre a noviembre, siendo mayo el mes más lluvioso. Los períodos secos van de diciembre a febrero y de agosto a septiembre, siendo enero el mes más seco 
[1]. Rabanal es un páramo semihúmedo a seco, de acuerdo con la clasificación climática Caldas-Lang, que se basa en la variación altitudinal de la temperatura y la efectividad de la precipitación [20]. La estructura del paisaje corresponde a un mosaico en el que predomina la vegetación herbácea, tanto en el páramo natural, como en las zonas afectadas por la actividad humana [1].

Los fragmentos de bosque altoandino presentes en el páramo de Rabanal corresponden a unas 6-7 ha con vegetación de hasta $12 \mathrm{~m}$ de altura en el estrato arbóreo, dominados por especies de las familias Melastomataceae, Lauraceae, Rubiaceae, Rosaceae, Ericaceae y Poaceae. Adyacentes a dichos fragmentos se encuentran áreas de páramo azonal con altos niveles freáticos de unas 10 ha dominadas por Espeletia murilloi y E. boyacensis, y por herbáceas como Puya goudotiana, P. trianae, Paepalanthus sp., Calamagrostis effusa, Valeriana pilosa, Arcytophyllum muticum y Eryngium humboldtii, y por el musgo $S p$ hagnum sp. Colindan con estos, zonas de pajonal ( $C$. effusa) en las que se encuentran inmersos el frailejón endémico, Espeletiopsis rabanalensis y E. argentea, así como arbustos de las familias Ericaceae y Asteraceae [21]. Dos humedales naturales, Laguna Verde y Laguna Cristal, con una extensión de 3,69 ha y 0,61 ha, respectivamente, hacen parte de la zona evaluada [1].

En el complejo predominan rocas sedimentarias de origen marino, que se formaron en el Cretáceo, junto con rocas terciarias de ambiente de transición marino-continental. Adicionalmente, y cubriendo estas secuencias, se encuentran rocas piroclásticas representadas por las cenizas volcánicas, depósitos cuaternarios de origen glaciar, lacustre, fluvial o aluvial. Sobre estos sustratos se han desarrollado suelos superficiales a profundos, bien drenados en las fuertes pendientes y pobremente drenados en las depresiones. La fertilidad de los suelos es baja, debido a la poca saturación de bases y a la alta acumulación de aluminio, lo cual genera fuerte acidez. El contenido de materia orgánica es alto, debido al lento proceso de descomposición y a la influencia de cenizas volcánicas provenientes de la cordillera Central [1].

\subsection{Técnicas de muestreo}

Se realizaron tres muestreos de siete días cada uno, entre febrero y agosto del 2014, abarcando así tanto el periodo seco como el periodo de máxima pluviosidad. Para el periodo seco se realizó un muestreo en el mes de febrero, y para la época lluviosa se realizaron dos muestreos, en los meses de abril y agosto. En cada uno fueron evaluadas dos estaciones similares en sus coberturas vegetales. Las coberturas vegetales muestreadas fueron Bosque (B), Borde Bosque-Frailejonal (B-F), Frailejonal (F) y Pastizal (P). En cada estación se instalaron cuatro transectos, uno por cobertura. Cada transecto consistió de cinco trampas separadas 30 metros entre sí, para un total de $150 \mathrm{~m}$. En total, para las dos estaciones se instalaron ocho transectos y 40 trampas por muestreo, y un total de 5760 horas efectivas de muestreo. En las estaciones los transectos estaban separados por $100 \mathrm{~m}$ de distancia [22] y las estaciones estaban separadas entre sí por $1 \mathrm{~km}$ aproximadamente (figura 1).

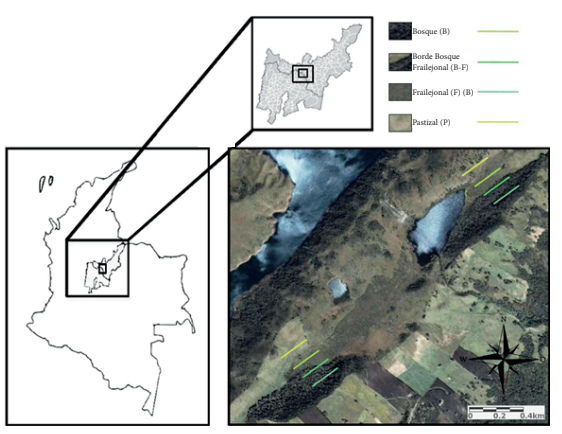

Figura 1. Área de estudio. Ubicación de los transectos realizados en las diferentes coberturas en el Páramo de Rabanal, Boyacá, Colombia (Tomado y modificado de Latorre et al. [23]).

\subsection{Técnicas de captura}

La captura de los individuos se realizó mediante trampas de caída estándar con atrayente, que constan de un vaso plástico de 16 onzas con 4 onzas de alcohol al 70\%, enterrado al ras del suelo, sobre el cual se instala un alambre en forma de " $U$ " invertida, en cuya cúspide se ancla un recipiente plástico que contiene el cebo; en éste caso, humanasa [22]. Las trampas fueron cebadas con 20 gramos de atrayente que era reemplazado cada 24 horas durante los siete días que duró cada muestreo. Los individuos colectados fueron preservados en alcohol al $70 \% \mathrm{y}$, posteriormente, montados en alfileres para ser depositados en la colección entomológica de la Universidad Pedagógica y Tecnológica de Colombia (UPTC, Tunja). La determinación taxonómica se realizó mediante las claves de Vaz-de-Mello et al. [24]. Para determinar el sexo de los individuos se 
realizó la disección de los genitales de cada uno, registrando como machos aquellos que presentaron edeago (aparato reproductor masculino) [31].

\subsection{Análisis de datos}

Se realizó un conteo de individuos para las coberturas vegetales muestreadas, hallando para cada una la abundancia relativa de Homocopris achamas. Se consideró la abundancia relativa como la cantidad de individuos de la especie encontrados en la cobertura, dividida por el número total de individuos capturados en el área total de muestreo. Para detectar diferencias estadísticas en las abundancias relativas de $H$. achamas para las coberturas estudiadas se utilizó la prueba no paramétrica de Kruskal-Wallis o Anova por rangos, debido a la anormalidad de los datos, en la que se tomó como tratamiento cada cobertura. Se realizó a posteriori la prueba de comparaciones múltiples HSD o Tukey para encontrar las coberturas vegetales que diferían de las demás. Estos análisis fueron realizados en el software estadístico SigmaPlot.

\section{Resultados}

Se registró una única especie de la familia Scarabaeidae: Homocopris achamas, de la cual se capturaron un total de 46 individuos ( $n=46$ ); un individuo fue capturado en la cobertura Bosque $(n=1)$, cinco en el borde Bosque-Frailejonal $(n=5), 22$ en el Frailejonal $(n=22)$ y 18 en el Pastizal $(n=18)$ (figura 2 ). De estos el $36.4 \%$ fueron hembras, y el $63.6 \%$, machos. La totalidad de los individuos fueron colectados en los meses mayo y julio durante la época lluviosa, mientras que en el muestreo realizado en agosto, durante la época seca, no se colectó ningún ejemplar.

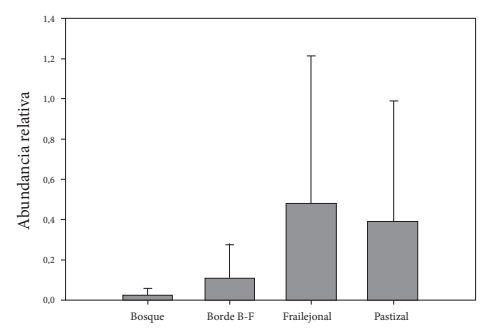

Figura 2. Abundancias relativas de Homocopris achamas en las diferentes coberturas vegetales presentes en el Páramo de Rabanal, Boyacá, Colombia.

Los resultados de la prueba de Kruskal-Wallis arrojaron diferencias significativas entre las abun- dancias relativas de las diferentes coberturas $(p=$ $0.0005 ; \alpha<0,05$ ). Las pruebas posthoc de Tukey no arrojan diferencias significativas entre las abundancias encontradas en Frailejonal y Pastizal, al igual que entre Bosque y Borde de Bosque; pero sí arrojan diferencias significativas entre Pastizal y Frailejonal con bosque y borde de bosque.

\section{Discusión}

La riqueza de especies de escarabajos coprófagos en el Páramo de Rabanal está representada por una única especie, y es considerada baja, en comparación con la que presentan localidades a menor altitud, en las que se reportan hasta 11 especies entre los 2800 y $2900 \mathrm{msnm}$ [25]; esto se debe al efecto que ejerce el rango altitudinal en la familia Scarabaidae, cuya riqueza de especies disminuye conforme aumenta la altitud [15, 26, 27]. No obstante, esta riqueza es similar a la encontrada por Martínez-Revelo \& Lopera [17], que reportan ocho especies en páramos de Nariño, de las cuales, tres fueron encontradas por encima de los 3000 m: Oruscatus opalescens Bates, 1870; Uroxys elongatus Harold, 1868 y Homocopris achamas Harold, 1867.

Homocopris achamas, cuyo género fue recientemente revalidado [28], fue la única especie encontrada en el páramo de Rabanal, registrando bajas abundancias $(n=46)$ en comparación con otros estudios realizados en bosques altoandinos a $2800 \mathrm{~m}$, en los que se encontraron mayores abundancias de esta especie con un esfuerzo de muestreo similar ( $\mathrm{n}=540 ; 3840$ horas de muestreo) [24]. Esto coincide con Martínez-Revelo \& Lopera [17], quienes sugieren que el límite altitudinal para esta especie son los $3620 \mathrm{~m}$, esperando menores abundancias conforme aumenta la altitud. La distribución en Colombia de H. achamas incluye los departamentos de Nariño, Valle del Cauca, Cundinamarca y Boyacá, desde los $2250 \mathrm{~m}$, restringida a bosques altoandinos y páramos [15-17].

La ausencia de $H$. achamas durante la época seca puede deberse a la diferencia en el esfuerzo de muestreo, ya que durante la época húmeda este esfuerzo fue dos veces mayor. Aunque Amat et al. [25] reportan su colecta durante todo el año en relictos de bosques altoandinos de la cordillera Oriental, es necesario realizar nuevos muestreos, con el fin de descartar que la ausencia reportada en Rabanal, 
sea debido a los cambios de humedad durante estas épocas o a alguna relación fenológica con el medioambiente de páramo.

La preferencia de $H$. achamas por el frailejonal, en donde se capturaron 22 individuos, puede ser explicada por la oferta de materia orgánica en descomposición presente en esta cobertura vegetal, que podría suponer algún tipo de recurso alimenticio, debido a la ausencia de grandes mamíferos que generen una oferta importante de estiércol [21]; además, el efecto de la necromasa impide que se alcancen temperaturas bajo cero durante la noche, que puedan provocar la congelación de los animales que viven allí $[3,10,29,30]$, convirtiendo este lugar en un hábitat potencial para estos escarabajos de hábito cavador. Sin embargo, esta es una hipótesis, ya que aún no se registran evidencias que demuestren este tipo de asociación.

Por otro lado, abundancias similares fueron encontradas en pastizal, con 18 individuos, lo cual puede deberse a la oferta del recurso que representa el estiércol vacuno. Aunque en los pastizales muestreados no se encontraron señales recientes de la presencia de algún tipo de ganado, es probable que estos insectos se desplacen desde otros pastizales aledaños, que distan unos $500 \mathrm{~m}$ de la ubicación de los transectos y que tienen presencia constante de ganado. Es probable que H. achamas use de manera similar los pastizales y frailejonales, debido a que en ambos lugares existe algún tipo de oferta de recurso y a la facilidad en el desplazamiento que ofrecen por ser zonas abiertas.

Homocopris achamas es considerado un escarabajo de potrero con la capacidad de penetrar en el bosque [25]; esto coincide con lo observado en el presente estudio, en donde las mayores abundancias de esta especie se presentaron en zonas abiertas $(n=40)$, con capturas esporádicas en el borde e interior de bosque $(n=6)$, sugiriendo un mínimo uso de los relictos boscosos de páramo por esta especie.

Martínez-Revelo \& Lopera [17] proponen que Homocopris achamas no es atraído por excremento humano ni carroña por encima de los $3000 \mathrm{~m}$ en los páramos de Nariño; sin embargo, en el páramo de Rabanal la totalidad de capturas se realizaron utilizando trampas de caída con humanasa como atrayente; esto sugiere que la eficacia del atrayente puede variar de acuerdo con las condiciones ambientales de los ecosistemas de alta montaña, que son particulares para cada páramo. Lo anterior, pone de manifiesto la necesidad de evaluar la eficacia de las trampas y el área de cobertura de los atrayentes, teniendo en cuenta las variables abióticas que puedan afectar de modo alguno la difusión de las sustancias atractivas para el escarabajo. Por otra parte, también es posible que las poblaciones de $H$. achamas de los páramos de Nariño sean mucho menores que las de Boyacá y Cundinamarca (Lopera, com. pers.), por lo cual se recomienda extender el muestreo a otros páramos colombianos con el fin de entender las diferencias y fluctuaciones de las poblaciones de esta especie en los ambientes de alta montaña.

Finalmente, es necesario ampliar los muestreos para la familia Scarabaeidae en los diferentes páramos de Colombia, con el fin de acercarse a la verdadera diversidad de escarabajos coprófagos, a sus dinámicas e interacciones con estos ecosistemas considerados estratégicos y de alta importancia ecológica, social y cultural.

\section{Agradecimientos}

Al Laboratorio de Entomología de la Universidad Pedagógica y Tecnológica de Colombia; al Museo de Historia Natural 'Luis Gonzalo Andrade' y al Herbario UPTC; al Grupo de Investigación Sistemática Biológica -SisBio- y a la profesora María Eugenia Morales Puentes, a Alejandro Lopera y a los evaluadores anónimos por sus valiosos comentarios en el enfoque del manuscrito; a los biólogos Naisla Manrique, Wilderson Medina, Juan Carlos Zabala, Claudia Hernández y Yudi Sissa, por su apoyo en campo; a Don Cristóbal, dueño de la finca; a la Dirección de Investigaciones -DIN-, al proyecto 1219 (Convenio 5211740, Ecopetrol-UPTC), a la UPTC, y a Colciencias por la financiación de este proyecto.

\section{Referencias}

[1] M. Morales, J. Otero, T. Van der Hammen, A. Torres, C. Cadena, C. Pedraza, N. Rodríguez, C. Franco, J. C. Betancur, E. Olaya, E. Posada y L. Cárdenas, Atlas de páramos de Colombia. Instituto de Investigación de Recursos Biológicos Alexander von Humboldt. Bogotá, D. C. 208.

[2] N. F. Alzate, "Insectos asociados a la necroma- 
sa de frailejón (Espeletia hartwegiana), en un páramo de Villamaría, Caldas", Agron., vol. 18, no. 1, pp. 59-68, 2010.

[3] G. Amat y O. Vargas, "Caracterización de microhábitats de la artropofauna en páramos del Parque Nacional Natural Chingaza Cundinamarca, Colombia”. Caldasia, vol. 16, no. 79, pp. 539-550, 1991.

[4] C. Bernal y C. Figueroa. "Estudio ecológico y comparativo de la entomofauna en un bosque altoandino y un páramo localizado en la región de Monserrate. Bogotá". Tesis Universidad Nacional de Colombia, 1980.

[5] O. Rangel y A. Bernal, "La entomofauna asociada a tres formaciones vegetales". Bol. Div. Depto. Bioi. U. Nal., vol. 1, no. 2, pp. 34-51, 1980.

[6] R. Herrera y F. Ruíz. "Algunos aspectos de la ecología y de los efectos inmediatos del fuego sobre la artropofauna asociada a Espeletia grandiflora $\mathrm{H}$. et B. Páramo de Monserrate, Bogotá". Tesis de grado. Universidad Nacional de Colombia. 1981.

[7] H. Sturm, "Zur Bodenfauna der andinen Paramoregion". Amazoniana, vol. 8, no. 1, pp. 129-147, 1983.

[8] H. Sturm y O. Rangel, Ecología de los páramos andinos. Instituto de Ciencias Naturales-Museo de Historia Natural. Biblioteca José Jerónimo Triana No. 9. Bogotá. Universidad Nacional de Colombia, 1985.

[9] R. H. B. Tobón, "Insectos de los páramos: maravillas en la coevolución entre plantas y animales" Boletín Cultural y Bibliográfico, vol. 23, no. 08, pp. 33-42, 1986.

[10] S. Sendoya y M. A. Bonilla, "La necromasa de Espeletia grandiflora como hábitat de la artropofauna de páramo". En: M. A. Bonilla. Estrategias adaptativas de plantas de páramo $y$ de bosque alto andino en la cordillera Oriental de Colombia. Bogotá: Unibiblios, p. 365, 2005.

[11] I. T. Morales-Castaño y G. D. Amat-García, "Diversidad de la artropofauna terrestre del páramo la Parada del Viento, cordillera Oriental, Cundinamarca-Colombia”. Boletín de la Sociedad Entomológica Aragonesa (S.E.A.), no. 51, pp. 211-216, 2012.
[12] M. A. Villalobos-M, "Nueva especie de mariposa (Lepidóptera: Nymphalidae: Satyrinae) para los andes colombianos". Boletín Científico Museo de Historia Natural, vol. 17, no. 1, pp. 268-275, 2013.

[13] C. A. Bota-Sierra, "A brief look at the Odonata from the Páramo ecosystems in Colombia, with the descriptions of Oxyallagma colombianum sp. nov. and Rhionaeschna caligo sp. nov. (Odonata: Coenagrionidae, Aeshnidae, Libellulidae)". Zootaxa, vol. 3856, no. 2, pp. 192-210, 2014.

[14] J. A. Noriega, E. Camero R, J. Arias-Buriticá, L. C. Pardo-Locarno, J. M. Montes, A. Acevedo, A. Esparza, B. Murcia-Ordóñez, H. García y C. Solís, "Grado de cobertura del muestreo de escarabajos coprófagos (Coleoptera: Scarabaeidae: Scarabaeinae) en Colombia". Rev. Biol. Trop., vol. 63, no. 1, pp. 97-125, 2015.

[15] F. Escobar, "Diversidad y distribución de los escarabajos del estiércol (Coleoptera: Scarabaeinae) de Colombia" (Pp. 197-201). En: F. Martín-Piera, J. J. Morrone y A. Melic (Eds.): Hacia un proyecto CYTED para el inventario y estimación de la diversidad entomológica de Iberoamérica PRIBES 2000. Monografías Tercer Milenio, vol. 1. Sociedad Entomológica Aragonesa, CYTED. Instituto de Investigación de Recursos Biológicos Alexander von Humboldt, p. 326, 2000.

[16] C. Medina, A. Lopera-Toro, A. Vítolo y B. Gill, "Escarabajos coprófagos (Coleóptera: Scarabaeidae: Scarabaeinae) de Colombia". Biota Colombiana, vol. 2, no. 2, pp. 131-144, 2001.

[17] D. E. Martínez-Revelo y A. Lopera-Toro, "Escarabajos coprófagos (Coleoptera: Scarabaeidae: Scarabaeinae) de los páramos del departamento de Nariño (Colombia). Biota Colombia$n a$, vol. 15, no. 1, pp. 62-72, 2014.

[18] F. Molano Rendón y C. A. Medina. "Especie nueva de Scybalocanthon (Coleoptera: Scarabaeinae: Canthonini) y descripción de la variación del órgano genital masculino". Revista Mexicana de Biodiversidad, no. 81, pp. 689699, 2010.

[19] F. Molano y D. A. Parrales, "Una nueva especie de Scybalocanthon, Martínez, 1948. (Coleoptera: Scarabaeidae) de los Andes de Colombia". 
Boletín Científico Centro de Museos Universidad de Caldas. En prensa, 2015.

[20] J. H. Gutiérrez, "Clasificaciones climáticas". Bogotá, Colombia: Instituto Colombiano de Hidrología, Meteorología y Adecuación de Tierras, HIMAT, 1991.

[21] W. A. Medina, D. C. Macana García, F. Sánchez, "Aves y mamíferos de bosque altoandinopáramo en el páramo de Rabanal (BoyacáColombia)". Ciencia en Desarrollo, vol. 6, no. 2, pp. 185-198, 2015.

[22] H. Villarreal., M. Álvarez, S. Córdoba, F. Escobar, G. Fagua, F. Gast, H. Mendoza, M. Ospina y A. M. Umaña, Manual de métodos para el desarrollo de inventarios de biodiversidad. Programa de inventarios de biodiversidad. Instituto de Investigación de Recursos Biológicos Alexander von Humboldt. Bogotá, Colombia, p. 236, 2004.

[23] I. T. Latorre-Beltrán, R. Novelo-Gutiérrez y M. E. Favila, "Diversidad genérica de Trichoptera (Insecta) en dos microcuencas del Páramo Rabanal (Cundinamarca-Boyacá, Colombia)", Revista de Biología Tropical, vol. 62, no. 2, pp. 97-110, 2014.

[24] F. Z. Vaz-de-mello, W. D. Edmonds, F. C. Ocampo y P. Schoolmeesters, "A multilingual key to the genera and subgenera of the subfamily Scarabaeinae of the New World (Coleoptera: Scarabaeidae)". Zootaxa, no. 2854, pp. 1-73 (Online edition). 2011. Disponible en: http://museum.unl.edu/research/entomology/ Guide/Scarabaeoidea/Scarabaeidae/Scarabaei nae/ScarabaeinaeKey2011.pdf

[25] G. Amat, A. Lopera y S. Amézquita, "Patrones de distribución de escarabajos coprófagos (Coleoptera: Scarabaeidae) en relictos de bosque altoandino, cordillera Oriental de Colombia". Caldasia, vol. 19, no. 1-2, pp. 191-204, 1997.

[26] M. Lobo, y G. Halffter, "Biogeographical and ecological factors affecting the altitudinal variation of mountainous communities of coprophagous beetles (Coleoptera, Scarabaeoidea): a comparative study". Annals of the Entomological Society of America, no. 93, pp. 115-126, 2000.

[27] F. Escobar, J. Lobo y G. Halffter. "Altitudinal variation of dung beetle (Scarabaeidae: Scarabaeinae) assemblages in the Colombian Andes". Global Ecology and Biogeography, no. 14, pp. 327-337, 2005.

[28] F. Z. Vaz-de-Mello, F. Génier y B. T. Smith, "Reclassification of Homocopris Burmeister as avalid genus to accommodate three species formerly in Dichotomius Hope (Scarabaeidae: Scarabaeinae: Coprini)". The Coleopterists Bulletin, vol. 64, no. 3, pp. 192, 2010.

[29] M. Monasterio, "Adaptative strategies of Espeletia in the Andean desert paramo". High Altitude Tropical Biogoegraphy. Oxford University Press. Oxford, 1986.

[30] H. Díaz, "Estudio de la comunidad de microartrópodos en la hojarasca en pie de Espeletia schultzii en el páramo desértico". Tesis de pregrado en Biología, Facultad de ciencias. Universidad de los Andes de Mérida. Venezuela, 1983.

[31] E. Manjarrés, F. Molano, "Piezas genitales masculinas como carácter taxonómico en escarabajos Coprófagos (Coleoptera: Scarabaeidae) Male genital parts as taxonomic character in coprophagous beetles (Coleoptera: Scarabaeidae)". Ciencia en Desarrollo, vol. 4, no. 2, pp. 39-47, 2013. 